03.3

\title{
Флуктуации плотности и температуры за фронтом ударной волны при воздействии стратифицированного источника энергии
}

\author{
(C) О.А. Азарова ${ }^{1}$, О.В. Кравченко ${ }^{1}$, Т.А. Лапушкина ${ }^{2,}$ А.В. Ерофреев ${ }^{2}$ \\ ${ }^{1}$ Вычислительный центр им. А.А. Дородницына Федерального исследовательского центра \\ „Информатика и управление“ РАН, Москва, Россия \\ ${ }^{2}$ Физико-технический институт им. А.Ф. Иофрфе РАН, Санкт-Петербург, Россия \\ ๑ E-mail: tanyusha@mail.ioffe.ru
}

Поступило в Редакцию 27 марта 2020 г.

В окончательной редакции 2 апреля 2020г.

Принято к публикации 2 апреля 2020г.

Для условий, полученных в экспериментах по взаимодействию ударной волны с ионизационно-неустойчивой плазмой, на основе полных уравнений Навье-Стокса проведено численное моделирование воздействия термически стратифицированного источника энергии на фронт ударной волны. Показано, что его искривление, регистрируемое на шлирен-картинах, связано с более высокой температурой центральных слоев в источнике, а исчезновение обусловлено множественным образованием неустойчивостей Рихтмайера-Мешкова, проявляющихся в поле плотности газа. Показано, что при перераспределении энергии источника в слои за фронтом ударной волны формируются локальные области с повышенной в несколько раз температурой газа (по сравнению со значениями для однородного источника).

Ключевые слова: ионизационная неустойчивость, ударные волны, стратифицированный источник энергии, уравнения Навье-Стокса.

DOI: 10.21883/PJTF.2020.13.49587.18327

Исследованиям возможности управления сверхзвуковыми/гиперзвуковыми потоками за счет удаленного энергетического воздействия в настоящее время посвящено большое количество работ [1-3]. В экспериментах [4] получен эффект ослабления отраженной ударной волны за счет взаимодействия с модифицированной плазмой пограничного слоя, которая создается параллельными узкими протяженными разрядными областями. В [5] показано, что причиной уменьшения сопротивления при комбинированном воздействии является изменение параметров потока, вызываемое дополнительным образованием нестационарных вихревых структур, связанных с проявлением неустойчивостей Рихтмайера-Мешкова. В [6] доказывается возможность плазменного воздействия на процесс обтекания тела при помощи короткоимпульсного газового разряда, который увеличивает температуру электронов, оставляя при этом неизменной температуру газа. В [7] на основе неравновесной модели двойного лазерного импульса установлено, что с увеличением степени ионизации происходит уменьшение силы сопротивления поверхности тела. Возможность воздействия на ударную волну однородной газоразрядной среды экспериментально исследована в [8]. Численно показано, что при прохождении ударной волны через область разряда происходит образование дополнительного контактного разрыва. В [9] показано, что воздействие ионизационно-неустойчивой плазмы вызывает неустойчивый характер фронтов разрывов, их деформацию и размытие вплоть до полного исчезновения при шлирен-визуализации.
В настоящей работе представлены экспериментальные результаты по получению ионизационно-неустойчивой газоразрядной плазмы и ее взаимодействию с ударной волной, а также проведено численное моделирование воздействия термически стратифицированного источника энергии на фронт ударной волны. Полученные результаты могут быть полезны в областях управления сверхзвуковыми потоками и обеспечения влияния на процессы поджига и перемешивания.

Задача исследования взаимодействия плоской ударной волны со слоистой по температуре газа средой может быть решена путем создания на пути распространения ударной волны области ионизационно-неустойчивого газового разряда. Экспериментально были получены условия создания такого разряда [9] и организованы разряды с ионизационными стратами разного масштаба и частоты следования. На рис. 1 представлены фотографии полученных разрядов с крупномасштабными (до 10 на длину разряда) (a) и мелкомасштабными (более 20 на длину разряда) (b) стратами, которые представляют собой области с повышенной скоростью ионизации [10], а следовательно, с повышенной концентрацией и температурой электронов. Поскольку в эксперименте наблюдаются стоячие страты, за время горения разряда (порядка $1 \mathrm{~min}$ ) в областях повышенной температуры электронов джоулев нагрев приводит также к увеличению температуры газа, создавая температурно-слоистую структуру среды. Как было показано в [9], взаимодействие ударной волны с числом Маха 5 с такой областью приводит к искривлению ударно-волновой структуры течения и в случае мелкомасштабных температурных 


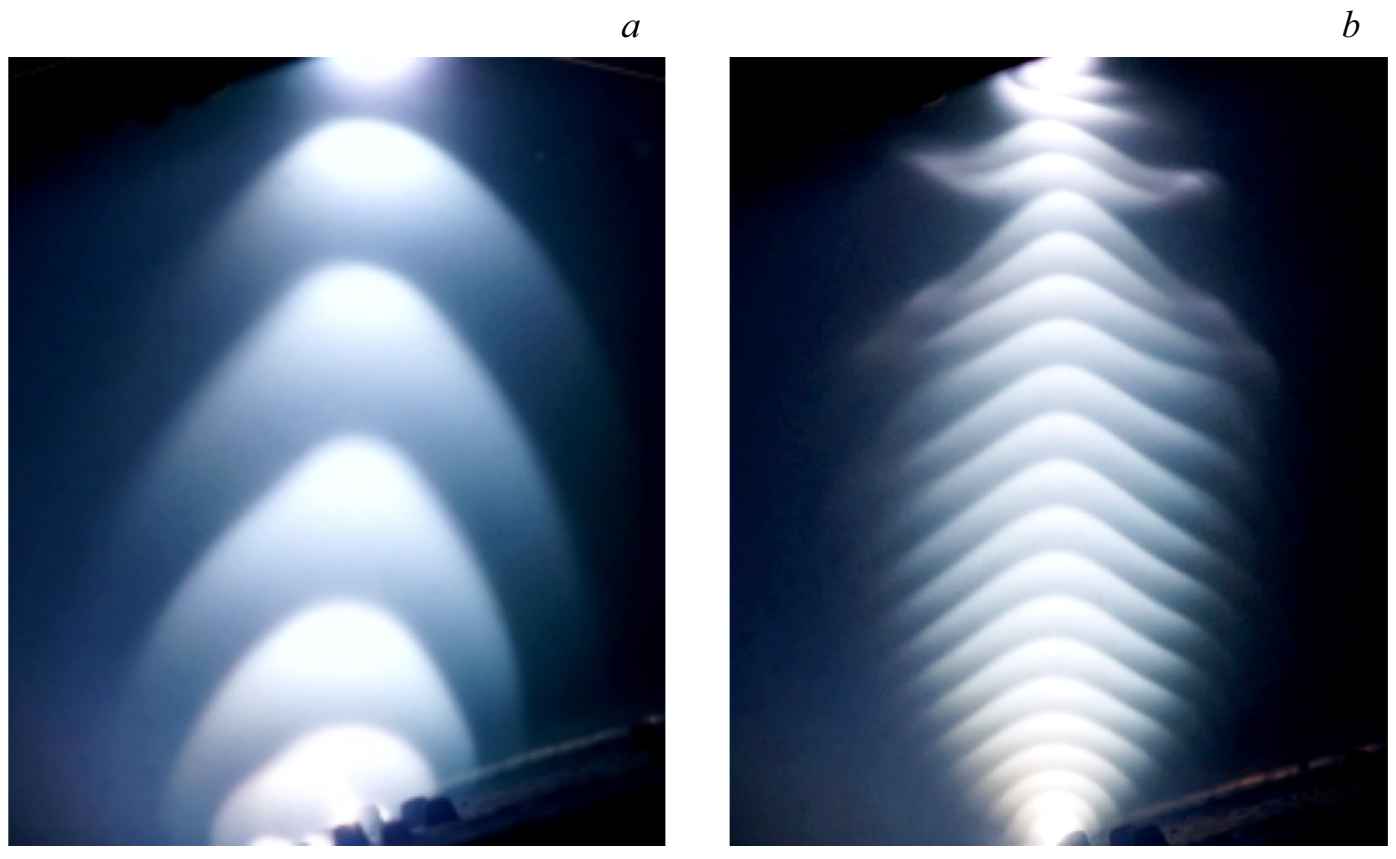

Рис. 1. Фотографии ионизационно-неустойчивого разряда. $a-$ крупномасштабные страты, $b-$ мелкомасштабные страты.

слоев может вызывать ее разрушение. В последнем случае шлирен-визуализация структуры течения показала отсутствие ударно-волновых градиентов плотности.

Численное моделирование основывалось на решении полной системы уравнений Навье-Стокса для вязкого теплопроводного газа (воздуха) [11]:

$$
\begin{gathered}
\frac{\partial \bar{U}}{\partial t}+\frac{\partial\left(\bar{F}+\bar{F}_{v}\right)}{\partial x}+\frac{\partial\left(\bar{G}+\bar{G}_{v}\right)}{\partial y}=0, \\
\bar{U}=\left(\begin{array}{c}
\rho \\
\rho u \\
\rho v \\
E
\end{array}\right), \bar{F}=\left(\begin{array}{c}
\rho u \\
p+\rho u^{2} \\
\rho u v \\
u(E+p)
\end{array}\right), \bar{G}=\left(\begin{array}{c}
\rho v \\
\rho u v \\
p+\rho v^{2} \\
v(E+p)
\end{array}\right), \\
\bar{F}_{v}=-\left(\begin{array}{c}
\mu / \operatorname{Re}\left(4 / 3 u_{x}-2 / 3 v_{y}\right) \\
\mu / \operatorname{Re}\left(v_{x}+u_{y}\right) \\
\mu \pi_{1} / \operatorname{Re}-(1 / N) k T_{x}
\end{array}\right), \\
0 \\
\bar{G}_{v}=-\left(\begin{array}{c}
\mu / \operatorname{Re}\left(v_{x}+u_{y}\right) \\
\mu / \operatorname{Re}\left(4 / 3 v_{y}-2 / 3 u_{x}\right) \\
\mu \pi_{2} / \operatorname{Re}-(1 / N) k T_{y}
\end{array}\right), \\
\pi_{1}=u\left(4 / 3 u_{x}-2 / 3 v_{y}\right)+v\left(v_{x}+u_{y}\right), \\
\pi_{2}=v\left(4 / 3 v_{y}-2 / 3 u_{x}\right)+u\left(v_{x}+u_{y}\right), \\
\rho\left(\varepsilon+0.5\left(u^{2}+v^{2}\right)\right), \quad N=\operatorname{RePr}(\gamma-1) / \gamma,
\end{gathered}
$$

где $\mathrm{Re}$ - число Рейнольдса, $\operatorname{Pr}$ - число Прандтля $(\operatorname{Pr}=0.703)$. Здесь $\rho, p, u, v-$ плотность газа, давление и $x$ - и $y$-компоненты скорости, $\varepsilon-$ удельная внутренняя энергия, $\varepsilon=p /(\rho(\gamma-1))$.
Для аппроксимации зависимости динамической вязкости $\mu$ от температуры использовался закон Сазерленда

$$
\mu=T^{1.5}\left(1+s_{1}\right) /\left(T+s_{1}\right)
$$

с константой $s_{1}=0.41(120 \mathrm{~K})$. Полагалось, что коэффициент теплопроводности $k$ зависит от температуры: $k=T^{0.5}$. Задача решалась в безразмерных переменных. Нормирующими значениями плотности и давления были значения, соответствующие экспериментальным условиям [9]: $\rho_{n}=\rho_{0}=0.01205 \mathrm{~kg} / \mathrm{m}^{3}$ и $p_{n}=p_{0}=1013.25 \mathrm{~Pa}$ соответственно. Нормирующее значение длины было равно расстоянию между горизонтальными стенками трубы: $l_{n}=0.05 \mathrm{~m}$. Число Рейнольдса равнялось $R e=9563[9]$. Рассматривалась газовая среда с эффективным показателем адиабаты $\gamma=1.2$, которое наилучшим образом приближает кривизну фронта ударной волны, полученную в эксперименте [8]. Это значение соответствует степени ионизации газовой среды 0.00015 и степени неравновесности 0.015 [12].

Стратифицированный источник энергии моделировался стационарной областью слоев разреженного газа одинаковой ширины, расположенной по всей ширине трубы на некотором расстоянии перед ударной волной. Внутри слоев (обозначенных индексом $i$ ) задавалась пониженная плотность газа $\rho_{i}=\alpha_{i} \rho_{0}, \alpha_{i}<1$. Остальные параметры задавались равными начальным параметрам потока внутри ударной трубы (обозначенным индексом 0): $p_{i}=p_{0}, u_{i}=0, v_{i}=0$. Таким образом, температура внутри слоев была повышенной по сравнению с начальной температурой газа в трубе: $T_{i}=\alpha_{i}^{-1} T_{0}$. На стенках трубы использовались условия прилипания $(u=0, v=0)$ и условия отсутствия потоков, нормальных к стенкам. Предполагалось, что горизонтальные 

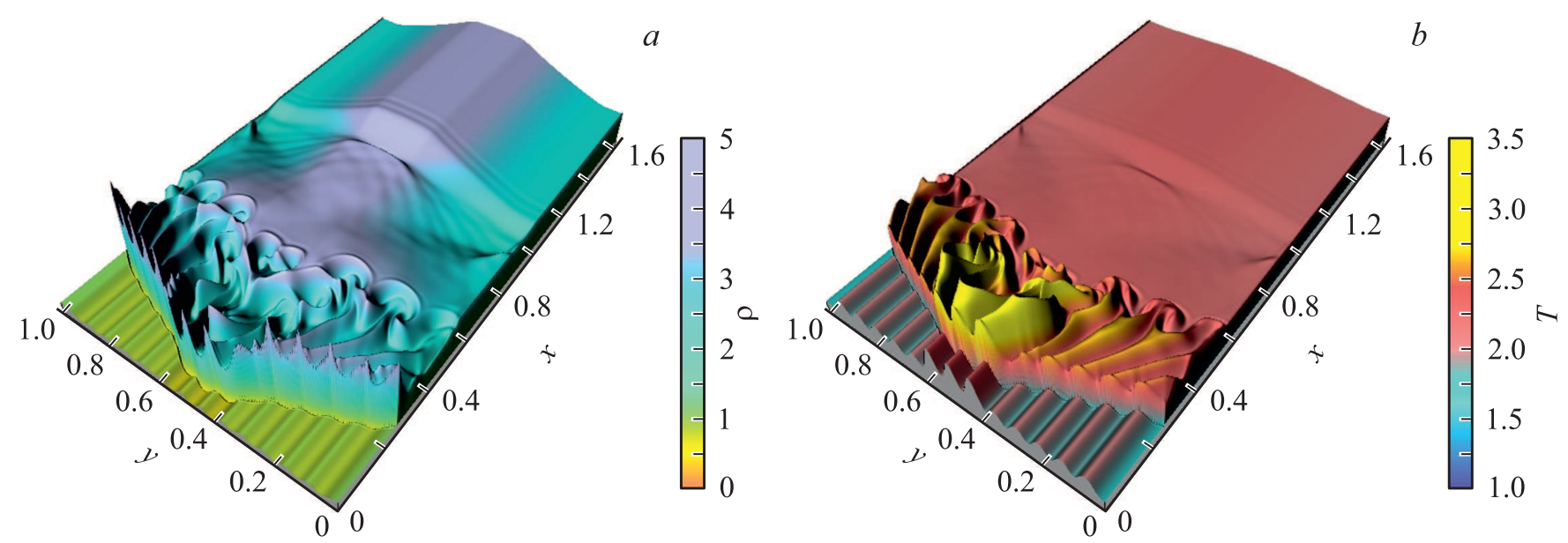

Рис. 2. Деформация фронта ударной волны при воздействии стратифицированного источника энергии, $\mathrm{M}=3$, $\alpha_{6}=\alpha_{7}=\alpha_{8}=0.45$, остальные $\alpha_{i}=0.7, h x=h y=0.001$. $a-$ поле плотности, $b$ - поле температуры.

стенки трубы остаются холодными (с температурой, равной $\left.T_{0}\right)$, а температура газа на стенках равняется температуре стенки.

В расчетах использовался программный код, основанный на комплексно-консервативных разностных схемах второго порядка аппроксимации [13]. Используется различный вид схем на границах и внутри трубы с сохранением свойства консервативности во всей расчетной области. Для повышения порядка аппроксимации использовались системы дифференциальных следствий системы (1) по $x$ и по $y$. Схемы являются явными, записываются на пятиточечном шаблоне (шаблоне схемы Лакса) и не содержат дополнительных членов искусственной вязкости и монотонизаторов. Использовались декартовы шахматные сетки с равными шагами по пространству $(h x=h y)$; на поперечный размер трубы приходилось $1000-2000$ узлов $\left(1.6 \cdot 10^{6}-6.4 \cdot 10^{6}\right.$ узлов в расчетной области с учетом среднего узла). В расчетах устойчивость схем обеспечивалась выполнением критерия Куранта-Фридрихса-Леви для выбора шага по времени с коэффициентом 0.2 (несмотря на присутствие диссипативных членов в системе (1)).

Координата ударной волны равнялась $x_{s w}=1.5$, правая граница источника энергии находилась в точке $x_{i}=1.4$ (между ними находились параметры невозмущенного потока). Ударная волна (с числом Маха М) движется справа налево и взаимодействует со стратифицированной областью источника. В случае однородной разогретой области, как можно заключить из решения задачи Римана, в результате взаимодействия образуются новые разрывы, ударная волна и контактный разрыв, движущиеся влево. В случае слоистого источника эти разрывы приобретают сложную форму, видоизменяясь за счет проявления неустойчивости Рихтмайера-Мешкова. Условия возникновения данной неустойчивости приведены в [14].

На рис. 2, $a$ представлено поле плотности (в виде поверхности), которое демонстрирует возможность де- формации фронта ударной волны, полученной в экспериментах, за счет более горячих центральных слоев в источнике энергии. Видно, что изогнутый фронт ударной волны качественно повторяет форму фронта волны, регистрируемого на шлирен-картине в эксперименте (см. рис. 8, $a$ в [9]). За фронтом ударной волны наблюдается образование сложных контактновихревых структур (грибовидных структур), связанное с множественным проявлением неустойчивостей Рихтмайера-Мешкова. Соответствующие поля температуры приведены на рис. 2, $b$. Видно, что за фронтом волны возникают локальные флуктуации температуры, значения в которых, как показали расчеты, могут в несколько раз превосходить значения температуры для однородного источника энергии (с равной величиной энергии импульса).

Шлирен-визуализация взаимодействия ударной волны с мелкомасштабными стратами демонстрирует размытие фронтов разрывов вплоть до полного исчезновения (см. рис. 9, $b, c$ в [9]). На рис. 3, $a$ приведено поле плотности, показывающее возможность почти полного размытия фронтов ударной волны и контактного разрыва за счет проявления неустойчивостей Рихтмайера-Мешкова. Так как шлирен-картины регистрируют градиенты плотности, полученные расчетные данные объясняют этот эффект. На полях температуры неустойчивость не проявляется (рис. $3, b$ ), однако поле температуры за фронтом волны также приобретает сложную форму: слоистую (с наличием повышенных значений) непосредственно за фронтом ударной волны и контактновихревую вдали от него, что обусловлено формированием грибовидных структур, сопутствующих проявлению неустойчивости Рихтмайера-Мешкова в полях плотности.

Таким образом, проведенные расчеты подтверждают, что полученные в эксперименте страты, продуцированные ионизационной неустойчивостью, характеризуются слоистым характером распределения температуры газа. 


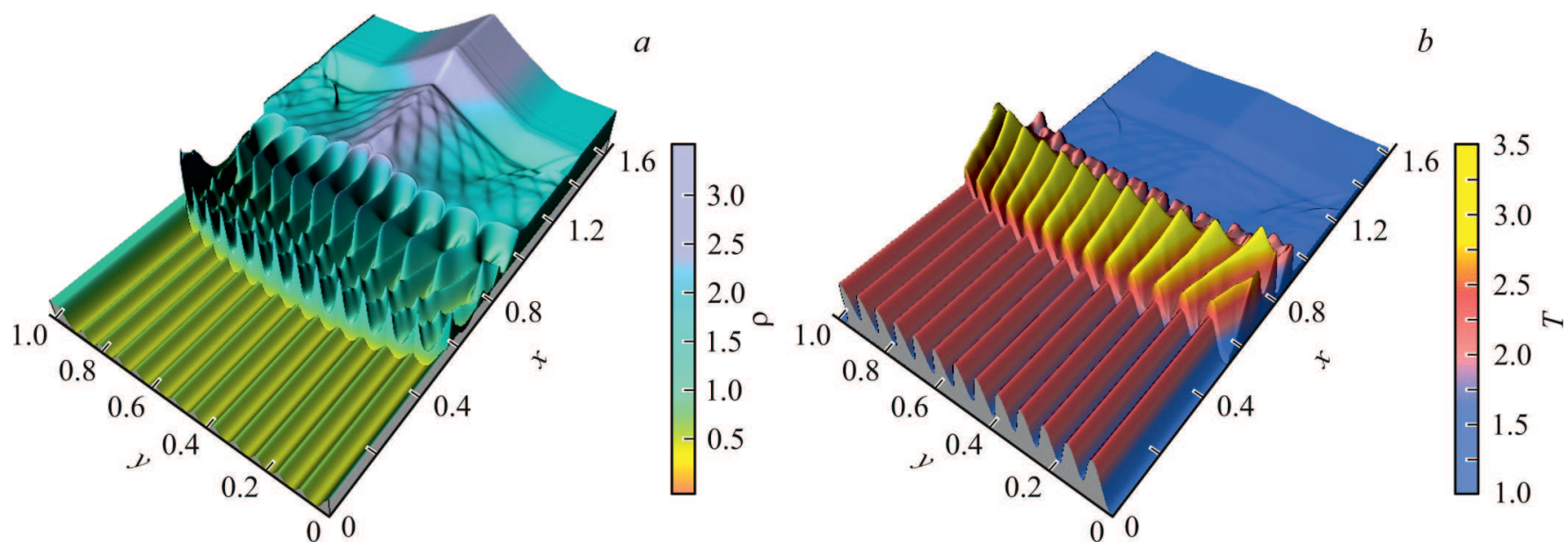

Рис. 3. Размытие фронта ударной волны при воздействии стратифицированного источника энергии, $\mathrm{M}=2$, все $\alpha_{i}=0.3$, $h x=h y=0.0005 . a-$ поле плотности, $b-$ поле температуры.

При этом воздействие страт на ударную волну ведет к искривлению ее фронта за счет более нагретых центральных слоев, а в некоторых случаях приводит к полному размытию фронта за счет множественного проявления неустойчивости Рихтмайера-Мешкова. В расчетах получено также, что поля температуры характеризуются повышенными локальными значениями за фронтом ударной волны по сравнению со значениями для однородного источника при такой же энергии импульса.

Результаты указывают на возможность применения стратифицированных источников энергии (лазерных, микроволновых, разрядных и комбинированных) для повышения эффективности воздействия на ударную волну в сфере контроля потока при сверхзвуковом обтекании тел, а также для интенсификации процессов поджига и перемешивания.

\section{Конфликт интересов}

Авторы заявляют, что у них нет конфликта интересов.

\section{Список литературы}

[1] Knight D.D. Energy deposition for high-speed flow control. Cambridge University Press, 2019. $450 \mathrm{p}$.

[2] Ahmed M.Y.M., Qin N. // Prog. Aerospace Sci. 2020. V. 112. P. 100585 .

[3] Russell A., Zare-Behtash H., Kontis K. // J. Electrostat. 2016. V. 4. P. $1-90$.

[4] Houpt A.W., Hedlund B.E., Leonov S.B., Ombrello T., Carter C.D. // 48th AIAA Plasmadynamics and Lasers Conf. Denver, Colorado, 2017. Paper AIAA-2017-3476. https://doi.org/10.2514/6.2017-3476

[5] Azarova O.A. // Aerospace. 2015. V. 2. P. 118-134.

[6] Азарова О.А., Ерофеев А.В., Лапушкина Т.А. // Письма в ЖТФ. 2017. Т. 43. В. 8. C. 93-101.
[7] Mahamud R., Hartman D.W., Tropina A.A. // J. Phys. D: Appl. Phys. 2020. In press.

[8] Лапушкина Т.А., Ерофеев А.В., Азарова О.А., Кравченко O.B. // ЖТФ. 2019. Т. 64. В. 1. C. 42-49.

[9] Lapushkina T.A., Erofeev A.V., Azarova O.A., Kravchenko O.B. // Aerospace Sci. Technol. 2019. V. 85. P. 347-358.

[10] Raizer Yu.P. Gas discharge physics. Springer, 1991. 463 p.

[11] Роуи П. Вычислительная газовая динамика. М.: Мир, 1980. $616 \mathrm{c}$.

[12] Kourtzanidis K., Raja L.L., Coumar S., Lago V. // 54th AIAA Aerospace Sciences Meeting. San Diego, California, 2016. Paper AIAA-2016-2157. https://doi.org/10.2514/6.2016-2157

[13] Азарова О.А. // ЖВМ и МФ. 2015. Т. 55. № 12. С. $2067-$ 2092.

[14] Hawley J.F., Zabusky N.J. // Phys. Rev. Lett. 1989. V. 63. P. 1241-1245. 